\title{
An identity involving Bernoulli numbers and the Stirling numbers of the second kind
}

\author{
Sumit Kumar Jha \\ International Institute of Information Technology \\ Hyderabad-500 032, India \\ e-mail: kumarjha.sumiteresearch.iit.ac.in
}

Received: 17 November 2019

Revised: 16 July 2020

Accepted: 16 July 2020

Abstract: Let $B_{n}$ denote the Bernoulli numbers, and $S(n, k)$ denote the Stirling numbers of the second kind. We prove the following identity

$$
B_{m+n}=\sum_{\substack{0 \leq k \leq n \\
0 \leq l \leq m}} \frac{(-1)^{k+l} k ! l ! S(n, k) S(m, l)}{(k+l+1)\left(\begin{array}{c}
k+l \\
l
\end{array}\right)} .
$$

To the best of our knowledge, the identity is new.

Keywords: Bernoulli numbers, Stirling numbers of the second kind, Riemann zeta function, Polylogarithm function.

2010 Mathematics Subject Classification: 11B68, 11B73.

\section{Introduction}

Definition 1.1. The Bernoulli numbers $B_{n}$ can be defined by the following generating function:

$$
\frac{t}{e^{t}-1}=\sum_{n \geq 0} \frac{B_{n} t^{n}}{n !}
$$

where $|t|<2 \pi$.

Definition 1.2. The Stirling number of the second kind, denoted by $S(n, m)$, is the number of ways of partitioning a set of $n$ elements into $m$ nonempty sets. 
The following formula expresses the Bernoulli numbers explicitly in terms of the Stirling numbers of the second kind $[3,5]$ :

$$
B_{n}=\sum_{k=0}^{n} \frac{(-1)^{k} k ! S(n, k)}{k+1} .
$$

In the following section, we prove a new identity for the Bernoulli numbers in terms of Stirling numbers of the second kind, of which the above formula is a special case.

\section{Main result}

Our main result is the following.

Theorem 2.1. For all non-negative integers $m, n$ we have

$$
B_{m+n}=\sum_{\substack{0 \leq k \leq n \\
0 \leq l \leq m}} \frac{(-1)^{k+l} k ! l ! S(n, k) S(m, l)}{(k+l+1)\left(\begin{array}{c}
k+l \\
l
\end{array}\right)} .
$$

Remark 2.2. Letting $m=0$ in the above equation gives us equation (1).

Proof. We start with the following integral from [2]

$$
(\alpha+\beta) \zeta(\alpha+\beta+1)=\int_{0}^{\infty} \frac{\operatorname{Li}_{\alpha}(-1 / t) \operatorname{Li}_{\beta}(-t)}{t} d t
$$

where $\zeta(\cdot)$ is the Riemann zeta function, and $\operatorname{Li}_{\alpha}(t)$ is the polylogarithm function.

Letting $\alpha=-m$, and $\beta=-n$ (non-negative integers) in the preceding equation, we get

$$
-(m+n) \zeta(1-m-n)=\int_{0}^{\infty} \frac{\mathrm{Li}_{-m}(-1 / t) \mathrm{Li}_{-n}(-t)}{t} d t
$$

The following representation from the note [4]

$$
\operatorname{Li}_{-n}(-t)=\sum_{k=0}^{n} k ! S(n, k)\left(\frac{1}{1+t}\right)^{k+1}(-t)^{k}
$$

allows us to evaluate the integral as

$$
\begin{aligned}
\int_{0}^{\infty} \frac{\mathrm{Li}_{-m}(-1 / t) \mathrm{Li}_{-n}(-t)}{t} d t & =\int_{0}^{\infty} \sum_{\substack{0 \leq k \leq n \\
0 \leq l \leq m}} \frac{(-1)^{k+l} k ! l ! S(n, k) S(m, l) t^{k}}{(1+t)^{k+l+2}} d t \\
& =\sum_{\substack{0 \leq k \leq n \\
0 \leq l \leq m}}(-1)^{k+l} k ! l ! S(n, k) S(m, l) \int_{0}^{\infty} \frac{t^{k}}{(1+t)^{k+l+2}} d t \\
& =\sum_{\substack{0 \leq k \leq n \\
0 \leq l \leq m}}(-1)^{k+l} k ! l ! S(n, k) S(m, l) \frac{\Gamma(k+1) \Gamma(l+1)}{\Gamma(k+l+2)} .
\end{aligned}
$$

Here $\Gamma(\cdot)$ is the gamma function. This completes the proof after noting the fact [1] that

$$
-(m+n) \cdot \zeta(1-m-n)=B_{m+n} .
$$




\section{References}

[1] Ahlfors, L. V. (1953). Complex Analysis, McGraw-Hill, New-York.

[2] Wolfram Research (2020). Polylogarithm: Integration (formula 10.08.21.0027.01). Available at: https://functions.wolfram.com/ZetaFunctionsandPoly logarithms/PolyLog/21/02/03/0005/

[3] Gould, H. W. (1972). Explicit formulas for Bernoulli numbers, Amer. Math. Monthly, $79(1), 44-51$.

[4] Landsburg, S. E. (2009). Stirling numbers and polylogarithms, preprint. Available at: http://www. landsburg.com/query.pdf.

[5] Qi, F., \& Guo, B. N. (2014). Alternative proofs of a formula for Bernoulli numbers in terms of Stirling numbers, Analysis (Berlin), 34 (3), 311-317. 